\title{
Estudio de la Reacción de Curado del Sistema Éter Diglicidílico del Bisfenol-A (DGEBA) y la Dietilentriamina (DETA) por Calorimetría Diferencial de Barrido
}

\author{
Filiberto González Garcia \\ Instituto de Macromoléculas, Universidade Federal do Rio de Janeiro \\ Norma Galego Fernández \\ Universidade Estadual do Norte Fluminense, Centro de Ciência e Tecnologia
}

Resumen: La reacción de curado del sistema compuesto por el éter diglicidílico del bisfenol-A (DGEBA) y la dietilentriamina (DETA) fue estudiada por calorimetría diferencial de barrido (DSC). Diferentes expresiones cinéticas fueron encontradas por experimentos isotérmicos y dinámicos las que justifican cambios mecanísticos con la temperatura de cura. La cinética de la reacción cumple un segundo orden cinético con una energía de activación de $90 \mathrm{~kJ} \mathrm{~mol}^{-1}$ a altas temperaturas (mecanismo no catalítico). La cinética de la reacción a bajas temperaturas manifestó la existencia de dos mecanismos competitivos, se determinaron las constantes de velocidades de reacción a 60 y $70^{\circ} \mathrm{C}$ y se encontró una energía de activación en el rango de 56.9 a $63.0 \mathrm{~kJ}$ $\mathrm{mol}^{-1}$, que está en perfecto acuerdo con la reportada a bajas temperaturas (mecanismo autocatalítico). También se muestra el diagrama de fases del sistema.

Palabras claves: Éter diglicidílico del bisfenol-A, dietilentriamina, reacción de curado, calorimetría diferencial de barrido.

The curing reaction of diglycidyl ether of bisphenol-A (DGEBA) and diethylentriamine system studied by differencial scanning calorimetry

\begin{abstract}
The curing reaction of diglycidyl ether of bisphenol-A (DGEBA) and diethylentriamine (DETA) system was studied by differential scanning calorimetry (DSC). Different kinetics expressions were found by isothermal and dynamic experiments that they justify mechanism changes with the temperature. The reaction follows a second order kinetics and has activation energy of $90 \mathrm{~kJ} \mathrm{~mol}^{-1}$ at high temperatures (non-catalytic mechanism). The kinetics of the reactions at low temperatures manifested the existence of two competitive mechanisms, the constants rate were determined at 60 and $70^{\circ} \mathrm{C}$ and has activation energy in the range of 56.9 to $63.0 \mathrm{~kJ} \mathrm{~mol}^{-1}$ that is in perfect agreement with the reported to low temperatures (autocatalytic mechanism ).
\end{abstract}

Keywords: Diglycidyl ether of bisphenol-A, diethylentriamine, curing reaction, differencial scanning calorimetry. 


\section{Introducción}

El mecanismo de reacción del sistema compuesto por la resina epoxídica tipo éter diglicidílico del bisfenol-A (DGEBA) y las diaminas ha sido extensamente estudiado por diferentes grupos de investigadores $^{[1-7]}$. El mecanismo de reacción de este sistema químico es bien conocido, del que se ha propuesto que ocurra por dos mecanismos, uno no catalítico y otro autocatalítico, según se muestra a continuación:

$$
\begin{aligned}
& \mathrm{E}+\mathrm{A}_{1} \rightarrow \mathrm{A}_{2}+(\mathrm{OH}) \\
& \mathrm{E}+\mathrm{A}_{2} \rightarrow \mathrm{A}_{3}+(\mathrm{OH}) \\
& \mathrm{E}+\mathrm{A}_{1}+(\mathrm{OH}) \rightarrow \mathrm{A}_{2}+2(\mathrm{OH}) \\
& \mathrm{E}+\mathrm{A}_{2}+(\mathrm{OH}) \rightarrow \mathrm{A}_{3}+2(\mathrm{OH})
\end{aligned}
$$

Donde: E es el grupo epoxídico; $\mathrm{OH}$ es el grupo hidroxilo inicial o formado por reacción entre los grupos epoxídico y amina; $\mathrm{A}_{1}, \mathrm{~A}_{2} \mathrm{y}_{3}$ corresponden al grupo amina primaria, secundaria y terciaria respectivamente. Las ecuaciones 1 y 2 corresponden al mecanismo no catalítico y las dos últimas ecuaciones 3 y 4 corresponden al mecanismo autocatalítico.

Se conoce que en la reacción de este sistema químico ocurren simultáneamente ambos mecanismos y que pueden ser más o menos importantes en dependencia de la temperatura de curado. La reacción también está caracterizada por la gelificación y la vitrificación. Mientras que por un lado la gelificación ocurre cuando el grado de reticulación alcanza un determinado valor, dependiendo únicamente de la funcionalidad, la estequiometria y la reactividad entre los grupos funcionales, por otro lado la vitrificación ocurre cuando la temperatura de curado es inferior a la $T_{g}$ máxima del sistema totalmente curado, o sea durante el curado isotérmico, a medida que aumenta la reticulación aumenta la $T_{g}$ hasta igualarse a la temperatura de curado, a partir de este instante la $\mathrm{T}_{\mathrm{g}}$ aumenta lentamente ya que el polímero se encuentra en el estado vítreo.

Numerosos investigadores han estudiado el sistema resina epoxídica-diamina con el objetivo de evaluar diferentes parámetros cinéticos ${ }^{[8-13]}$. Este trabajo tiene la finalidad de estudiar por calorimetría diferencial de barrido (DSC) la reacción del sistema constituido por el éter diglicidílico del bisfenol-A (DGEBA) y la dietilentriamina (DETA) con el objetivo de obtener información mecanística, evaluar algunos parámetros cinéticos además de obtener información cuantitativa por experimentos dinámicos del punto de equivalencia del sistema.

\section{Experimental}

\section{Materiales}

La resina epoxídica utilizada fue el éter diglicidílico del bisfenol-A(DGEBA), denominada Araldita GY-250, suministrado por la CIBA-GEIGY, la que fue deshidratada a vacío a $80^{\circ} \mathrm{C}$ durante 4 horas antes de su empleo. El equivalente epoxídico de la resina fue de 192g/eq., determinado por método potenciométrico ${ }^{[14]}$.

El endurecedor utilizado fue la dietilentriamina (DETA) suministrada por la CIBA-GEIGY, la que fue deshidratada sobre tamices moleculares antes de su empleo.

\section{Calorimetría Diferencial de Barrido (DSC).}

Los experimentos fueron realizados en un equipo Mettler DSC 30. Los experimentos dinámicos e isotérmicos fueron realizados, utilizando atmósfera de nitrógeno y empleando entre 5 y $10 \mathrm{mg}$ de la mezcla binaria DGEBA/DETA preparada antes de realizar cada experimento.

En los experimentos dinámicos fueron realizados barridos desde -50 a $200^{\circ} \mathrm{C}$ empleando diferentes concentraciones entre los grupos funcionales y una velocidad de calentamiento de $10^{\circ} \mathrm{C} \mathrm{min}^{-1}$, además se hicieron experimentos empleando cantidades equivalentes (e/e) entre los grupos funcionales efectuando cambios en la velocidad de calentamiento de 2.5 a $20^{\circ} \mathrm{C} \mathrm{min}^{-1}$.

Los experimentos isotérmicos fueron realizados empleando cantidades equivalentes (e/e) entre los grupos funcionales y un rango de temperaturas de 55 a $70^{\circ} \mathrm{C}$, determinándose la conversión final para cada temperatura estudiada a partir de un experimento dinámico posterior al isotérmico, desde -30 a $200^{\circ} \mathrm{C}$ utilizando una velocidad de calentamiento de $10^{\circ} \mathrm{C} \mathrm{min}^{-1}$.

\section{Resultados y Discusión}

\section{Determinación del punto de equivalencia}

La determinación de este parámetro es de vital importancia cuando se utiliza un sistema comercial de dos componentes (resina y endurecedor) del que se desconoce la concentración de grupos funcionales 
en uno de ellos y se desea utilizar relación estequiométrica entre grupos funcionales. Este parámetro fue determinado por experimentos dinámicos a una velocidad de calentamiento de $10^{\circ} \mathrm{C} \mathrm{min}^{-}$ ${ }^{1}$ empleando diferentes concentraciones ( $\%$ en peso) de dietielentriamina (DETA) con el objetivo de provocar cambios en el calor de reacción ${ }^{[15]}$. El punto de equivalencia fue calculado a partir de la ecuación 5 que se muestra a continuación:

Punto de equivalencia $=\left(100 \Delta \mathrm{H}_{\mathrm{c}}\right) /\left(\Delta \mathrm{H}_{\mathrm{b}}+\Delta \mathrm{H}_{\mathrm{c}}\right)$

Los valores correspondientes de $\Delta \mathrm{H}_{\mathrm{b}} \mathrm{y} \Delta \mathrm{H}_{\mathrm{c}}$ fueron determinados por extrapolación del intercepto entre las dos rectas, que se muestran en las Figuras $1 \mathrm{a}$ y $1 \mathrm{~b}$ respectivamente.

El punto de equivalencia corresponde con $10 \%$ (en peso) de DETA. Este valor está en perfecto acuerdo con el valor teórico de $9,47 \%$, calculado a partir del valor teórico de la DETA $\left(20,6 \mathrm{~g} \mathrm{eq}^{-1}\right)$ y está en perfecto acuerdo también con el equivalente epoxídico de la resina utilizada (192 $\mathrm{g} \mathrm{eq}^{-1}$ ) reportado por el suministrador y por el obtenido por un método espectroscópico $\left(\mathrm{RMN}-\mathrm{H}^{1}\right)$ desarrollado en nuestro laboratorio ${ }^{[16]}$.
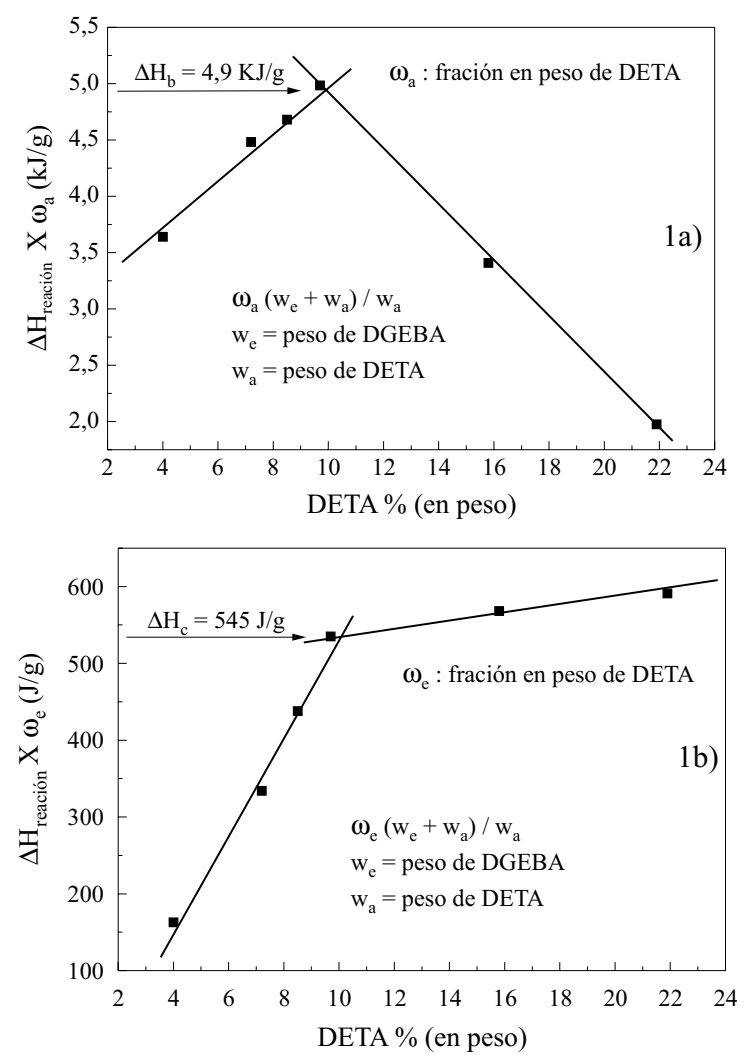

Figura 1. Determinación del punto de equivalencia del sistema DGEBA -DETA.

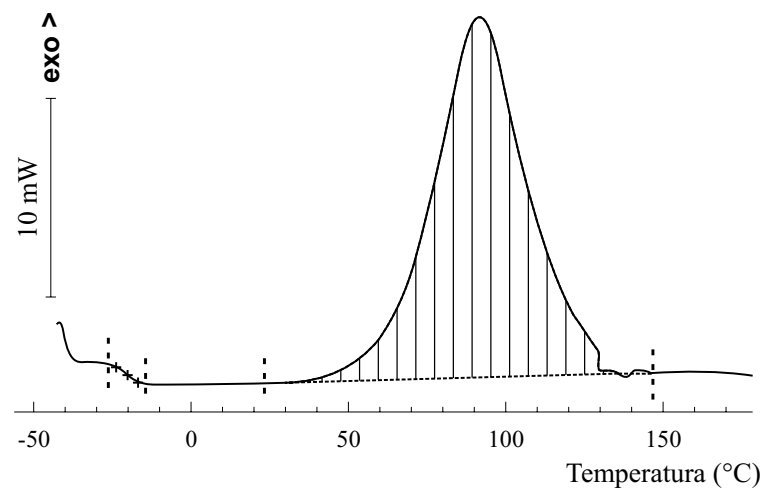

Figura 2. Termograma típico de los experimentos dinámicos del sistema DGEBA-DETA.

\section{Experimentos Dinámicos}

La Figura 2 muestra la forma típica de los termogramas de los experimentos dinámicos realizados empleando cantidades equivalentes (e/e) entre los grupos funcionales para estudiar la reacción de curado del sistema DGEBA-DETA.

La cinética de la reacción de curado fue obtenida a partir de los datos calorimétricos de 5 replicas de experimentos realizando barridos desde -50 a $200^{\circ} \mathrm{C}$ y una velocidad de calentamiento de $10^{\circ} \mathrm{C} \mathrm{min}^{-1}$. Los datos fueron procesados utilizado el clásico método de Barrett ${ }^{[17]}$, considerando que la cinética de la reacción cumple un comportamiento cinético de orden "n". Los resultados evidenciaron que el ajuste más satisfactorio fue para $n=2\left(R^{2}=0.995\right)$. La ecuación obtenida fue la siguiente:

$$
\mathrm{dX} / \mathrm{dt}=4.7 \times 10^{12} \mathrm{e}^{(-\mathrm{Ea} / \mathrm{RT})}(1-\mathrm{X})^{2}
$$

Donde $\mathrm{X}$ es la conversión $\left(\mathrm{X}=\mathrm{DH} / \mathrm{DH}_{\mathrm{T}}\right)$. Los resultados de éstos experimentos demuestran que la reacción tiene un comportamiento cinético de segundo orden con una energía de activación de 90.7 $\mathrm{kJ} \mathrm{mol}^{-1}$. Este valor de energía de activación está en el rango reportado en la literatura (80-105 kJ $\mathrm{mol}^{-1}$ ) para la energía de activación del mecanismo no catalítico ${ }^{[1-3]}$.

Información cinética del sistema fue también obtenida a partir de experimentos dinámicos variando la velocidad de calentamiento (q) para estudiar su relación con el desplazamiento de la temperatura del pico $\left(\mathrm{T}_{\mathrm{p}}\right)$ exotérmico correspondiente a la reacción de curado del sistema. Los datos experimentales fueron analizados por dos métodos: por el método de Ozawa y por el método de Kissinger ${ }^{[1,6]}$. Los resultados se muestran en la Figura 3. En este caso la ener- 


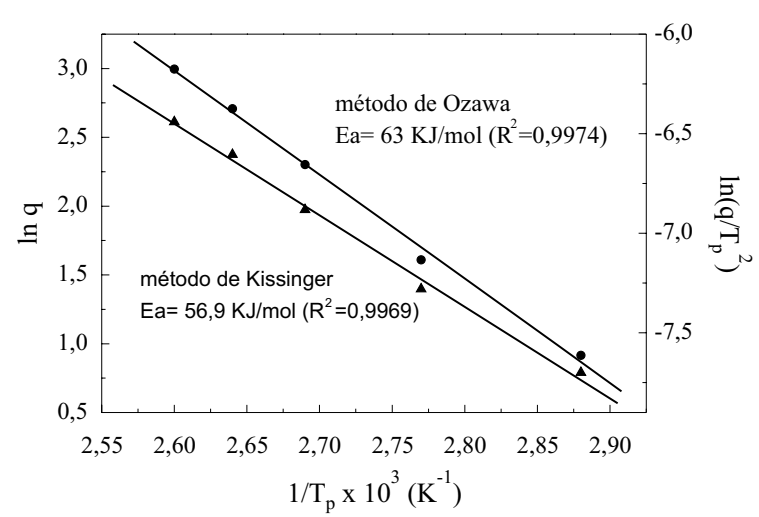

Figura 3. Experimentos dinámicos a diferentes velocidades de calentamiento: lnq x $1 / \mathrm{T}_{\mathrm{p}}$ - método de ozawa; $\ln \left(\mathrm{q} / \mathrm{T}_{\mathrm{p}}{ }^{2}\right)$ x $1 / \mathrm{T}_{\mathrm{p}}$ - método de kissinger

gía de activación obtenida está en el rango de 56.9 a $63.0 \mathrm{~kJ} \mathrm{~mol}^{-1}$, la que está en perfecto acuerdo con la energía de activación reportada para el mecanismo autocatalítico.

\section{Experimentos /sotérmicos:}

La Figura 4 muestra los termogramas de los experimentos isotérmicos realizados empleando cantidades equivalentes (e/e) entre los grupos funcionales para el sistema DGEBA-DETA. A partir de los datos calorimétricos fueron obtenidos los tiempos de curado $\left(t_{c}\right)$ para cada temperatura estudiada. Fue considerado como tiempo de curado $\left(\mathrm{t}_{\mathrm{c}}\right)$, el tiempo necesario para obtener una línea base en la curva calorimétrica. $\mathrm{El}$ inverso del tiempo de curado fue relacionado con la velocidad de reacción.

La Figura 5 muestra el logaritmo natural del inverso del tiempo de curado $\left(\mathrm{t}_{\mathrm{c}}\right)$ como una función del inverso de la temperatura (gráfico de Arrhenius). En la Figura 5 se observa claramente que existen dos líneas con diferente pendiente. Este resultado es fuerte evidencia de que no existe un comportamiento

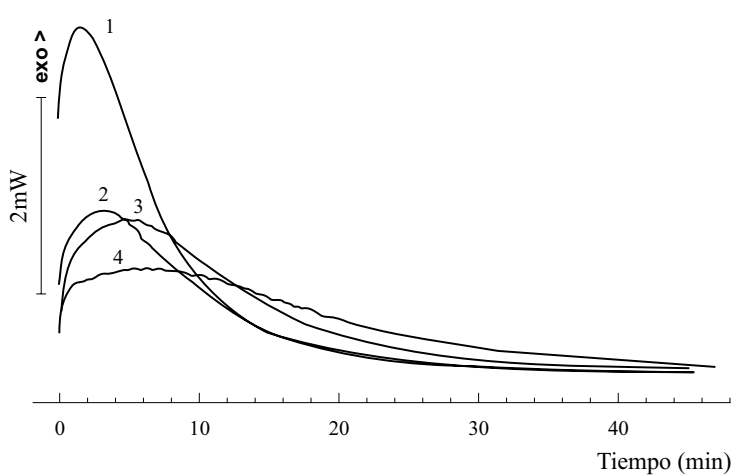

Figura 4. Termogramas de los experimentos isotérmicos del sistema DGEBA-DETA. $1.70^{\circ} \mathrm{C} ; 2.65^{\circ} \mathrm{C} ; 3.60^{\circ} \mathrm{C}$ y $4.55^{\circ} \mathrm{C}$.

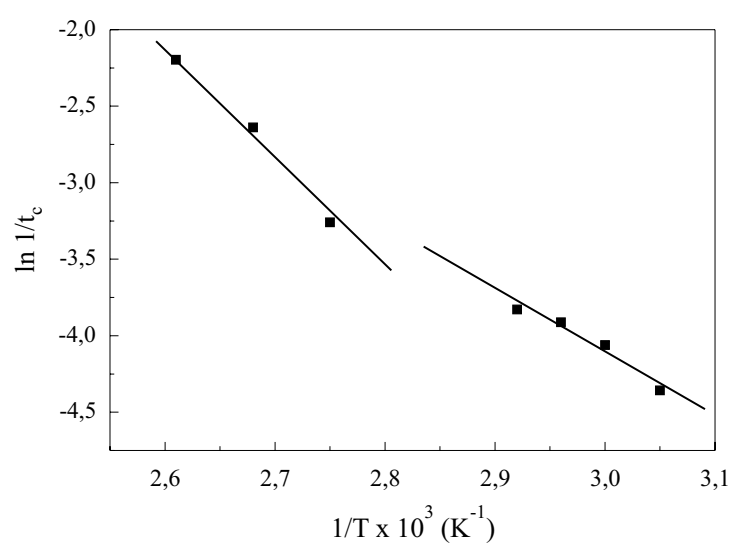

Figura 5. Gráfico de Arrhenius. Velocidad de reacción a diferentes temperaturas.

cinético único en todo el rango de temperaturas estudiadas, lo que está en perfecto acuerdo con que existen dos mecanismos competitivos en todo el rango de temperaturas. A baja temperatura, predomina el mecanismo autocatalítico (baja energía de activación) y a alta temperatura es más importante el mecanismo no catalítico (alta energía de activación).

Para evaluar las constantes cinéticas de cada mecanismo, fue considerado que:

- No existen efectos de sustitución entre los diferentes hidrógenos amínicos presentes en la dietilentriamina (DETA). O sea, existe igual reactividad, entre los grupos epoxídicos y los grupos aminas primaria o secundaria, iniciales o formados durante la reacción.

- La reacción tiene lugar por dos mecanismos competitivos; un mecanismo catalizado por los grupos hidroxilos inicialmente presentes en la resina epoxídica o los formados durante la reacción, y el otro mecanismo no catalítico que ocurre en todo el rango de temperaturas.

La ecuación utilizada fue la siguiente ${ }^{[1,3]}$ :

$$
\left.(\mathrm{dX} / \mathrm{dt}) /(1-\mathrm{X})^{2}=\left(\mathrm{k}_{\mathrm{a}}+\mathrm{k}_{\mathrm{c}}[\mathrm{OH}]_{\mathrm{o}}+\mathrm{k}_{\mathrm{c}}[\mathrm{e}]_{\mathrm{o}} \mathrm{X}\right)[\mathrm{e}]_{\mathrm{o}} 7\right)
$$

Donde: $\mathrm{k}_{\mathrm{a}}$ es la constante de velocidad del mecanismo no catalítico; $k_{c}$ es la constante de velocidad del mecanismo autocatalítico; $[\mathrm{OH}]_{\mathrm{o}}$ es la concentración inicial de grupos hidroxilos y $[\mathrm{e}]_{\mathrm{o}}$ es la concentración inicial de grupos epoxídicos.

Los datos calorimétricos de los experimentos isotérmicos a 60 y $70^{\circ} \mathrm{C}$ fueron procesados a partir de la ecuación 7; hasta una conversión aproximadamente de $\mathrm{X}=0,5$, los resultados del ajuste lineal del grafico de $(\mathrm{dX} / \mathrm{dt}) /(1-\mathrm{X})^{2}$ como función de $\mathrm{X}$, se muestran en la Tabla 1. Es importante destacar que en todo el ran- 
Tabla 1. Parámetros cinéticos del sistema DGEBA-DETA

\begin{tabular}{cccccc}
\hline $\mathbf{T}\left({ }^{\circ} \mathbf{C}\right)$ & $\mathbf{B}$ & $\mathbf{m}$ & $\begin{array}{c}\mathbf{k}_{\mathbf{a}} \\
(\mathbf{L} / \mathbf{e q ~} \mathbf{~ m i n}) \mathbf{x}\end{array} \mathbf{1 0}^{\mathbf{3}}$ & $\begin{array}{c}\mathbf{k}_{\mathbf{c}} \\
\left(\mathbf{L}^{2} / \mathbf{e q}^{2} \mathbf{m i n}\right) \mathbf{x} \mathbf{1 0}^{\mathbf{3}}\end{array}$ \\
\hline 60 & 0.017 & 0.105 & 0.35 & 3.39 \\
70 & 0.049 & 0.121 & 5.56 & 3.91 \\
\hline b: intersecto & m: pendiente & {$[\mathrm{OH}]_{\mathrm{o}}=0.83 \mathrm{eq} / \mathrm{L}$} & {$[\mathrm{e}]_{\mathrm{o}}=5.56 \mathrm{eq} / \mathrm{L}$}
\end{tabular}

go de conversión no se cumplió este comportamiento lineal; a valores superiores $(X>0,5)$ fue observada una desviación negativa. Este comportamiento puede ser explicado debido a que hasta aproximadamente $\mathrm{X}=$ 0,5 el mecanismo autocatalítico es más importante que el mecanismo no catalítico y aproximadamente poco después de $X=0,5$, ocurre la gelificación del sistema y como consecuencia ocurre una rápida disminución de la velocidad de reacción, posiblemente debido a problemas de difusión que dificultan la formación del complejo de transición entre los grupos epoxídicos-amina-hidroxilo en el mecanismo autocatalítico. Este comportamiento también fue encontrado anteriormente para otros sistemas de resina epoxídica-diamina ${ }^{[1-3]}$. Los valores de la Tabla 1, demuestran claramente que a $60^{\circ} \mathrm{C}$, la constante de velocidad del mecanismo autocatalítico $\left(\mathrm{k}_{\mathrm{c}}\right)$ es superior a la constante de velocidad del mecanismo no catalítico $\left(\mathrm{k}_{\mathrm{a}}\right)$ y que ocurre lo contrario a $70^{\circ} \mathrm{C}$. Estos resultados están en perfecto acuerdo con la ocurrencia simultánea de ambos mecanismos en la reacción del sistema DGEBA-DETA y que a temperaturas más elevadas la contribución del mecanismo no catalítico sea más importante que el autocatalítico.

En la Tabla 2, se muestran de modo comparativo los valores de la constante de velocidad del mecanismo autocatalítico $\left(\mathrm{k}_{\mathrm{c}}\right)$ a 60 y $70^{\circ} \mathrm{C}$ para el sistema estudiado, con los valores obtenidos por dos autores diferentes para un sistema similar, con la etilendiamina (EDA). Los valores mostrados en la Tabla 2 demuestran claramente que los valores de $\mathrm{k}_{\mathrm{c}}$ del sistema DGEBA-DETA son superiores a los valores del sistema DGEBA-EDA. Este efecto puede ser consecuencia de la presencia de la amina secundaria en la DETA.

La Figura 6 muestra la forma típica de los termogramas después de cada experimento isotérmico

Tabla 2. Valores comparativos de $\mathrm{k}_{\mathrm{c}}\left(\mathrm{L}^{2} / \mathrm{mol}^{2} \mathrm{~s}\right) \times 10^{4}$.

\begin{tabular}{cccc}
\hline Temperatura $\left({ }^{\circ} \mathbf{C}\right)$ & DETA & EDA $^{[3]}$ & EDA $^{[18]}$ \\
\hline 60 & 2.26 & 1.35 & 1.10 \\
70 & 2.60 & 2.50 & 1.90 \\
\hline
\end{tabular}

EDA: etilendiamina seguido de otro experimento dinámico, los que demuestran claramente el inicio de la vitrificación que ocurre después del experimento isotérmico y se manifiesta por la presencia de un calor residual, después de realizar el experimento dinámico. La conversión final alcanzada a diferentes temperaturas de curado, fue

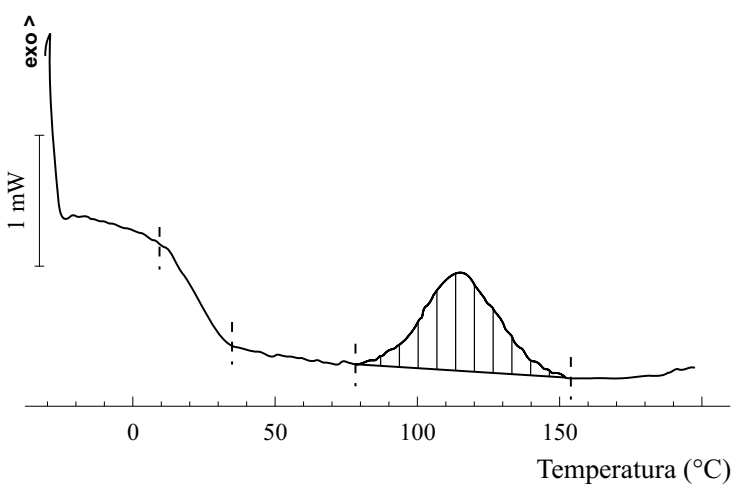

Figura 6. Calor residual. Termograma típico después del experimento isotérmico seguido de otro experimento dinámico.

calculada sumando el calor relativo de ambos experimentos sucesivos, o sea la suma del calor en el experimento isotérmico y el calor residual obtenido en el experimento dinámico posterior. Las conversiones finales así obtenidas fueron graficadas como una función de la temperatura de curado. Fueron incluidos en el grafico, los valores de $\mathrm{T}_{\mathrm{g}}$ máxima y de $\mathrm{T}_{\mathrm{g}}$ inicial del sistema obtenidos a partir de dos experimentos dinámicos sucesivos y un experimento dinámico respectivamente. La conversión final a diferentes temperaturas de curado está asociada con la vitrificación, esto se muestra en el diagrama de fases de la Figura 7. La curva de vitrificación se incrementa hasta el valor de Tg máxima a una conversión $=1\left(\mathrm{~T}_{\mathrm{g}}\right.$ máxima $\left.=110^{\circ} \mathrm{C}\right)$. Las fases pueden ser identificadas a partir de conocer la conversión donde ocurre la gelificación.

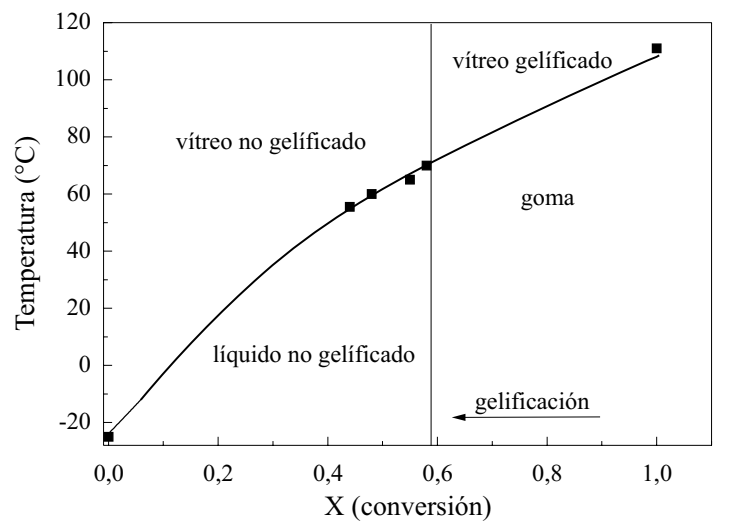

Figura 7. Diagrama de fases del sistema DGEBA-DETA. 


\section{Conclusiones}

El método realizado por experimentos dinámicos para determinar el punto de equivalencia del sistema DGEBA-DETA, llega a buenos resultados.

El análisis de la reacción de curado del sistema DGEBA-DETA mostró los siguientes hechos:

Por los experimentos dinámicos se encontró que la reacción de curado sigue un mecanismo no catalítico, con un comportamiento cinético de segundo orden y una energía de activación de $90 \mathrm{~kJ} \mathrm{~mol}^{-1}$. Estos experimentos están en perfecto acuerdo con lo reportado para el rango de altas temperaturas. En este caso el mecanismo autocatalítico no es una alternativa válida.

Por los experimentos isotérmicos se encontró que la reacción de curado ocurre por dos mecanismos competitivos. Las constantes cinéticas evaluadas fueron comparadas con otras de la literatura. Los métodos de Ozawa y de Kissinger fueron utilizados para evaluar la energía de activación del proceso. El rango obtenido (56.9-63.0 $\left.\mathrm{kJ} \mathrm{mol}^{-1}\right)$ está en perfecto acuerdo con la contribución del mecanismo autocatalítico que es más importante a bajas temperaturas.

Diferentes expresiones cinéticas fueron obtenidas por experimentos isotérmicos y dinámicos explicables debido a los cambios mecanísticos con la temperatura.

\section{Referencias Bibliográficas}

1. Barton, J. M. -Adv. Polym. Sci. 72, p.112 (1985).

2. Rozenberg, B. A. -Adv. Polym. Sci. 73, p.115 (1986).

3. Riccardi, C. C.; Adabbo, H. E. \& Williams R. J. J. - J. Appl. Polym. Sci. 29, p.2481 (1984).

4. Do Hyun Kim \& Sung Chul Kim -Polym. Bull.18, p.533 (1987).
5. Laza, J. M.; Julian, C. A.; Larrauri, E.; Rodríguez, M. \& León, L. M. -Polym. 40, p.35 (1998).

6. Carrozzino, S.; Levita, G.; Rolla, P. \& Tombari, E. Polym. Eng. Sci. 30, p.366 (1990).

7. Vyazorkin, S. \& Shinazzuoli N. - Macromolecules 29, p.1867 (1996).

8. Serier, A.; Pascualt, J. P. \& My, L. T. -J. Polym. Sci. Part A: Polym. Chem. 29, p.209 (1991).

9. Sabra, A.; Pascualt, J. P. \& Seytre, G. - J. Appl. Polym. Sci. 32, p.5147 (1986).

10. Mijovic, J.; Fichvain, A. \& Wijaya, J. - Macromolecules 25, p.979 (1992).

11. Riccardi, C. C. \& Williams R. J. J. - J. Appl. Polym. Sci.32, p.3445 (1986).

12. Núñez, L.; Fraga, F.; Fraga, L. \& Castro, A. - J. Appl. Polym. Sci. 63, p.635 (1997).

13. De Miranda, M. I. G.; Tomedi, C.; Bica, C. I. D. \& Samios, D. - Polym. 38, p.1017 (1997).

14. Lee H., \& Neville K., - "Handbook of Epoxy Resins", in: Characterization of Uncured Epoxy Resins, cap. 4, pag. 18, McGraw Hill, New York 1967

15. Eloundou, J. P.; Feve, M.; Hanan, D. \& Pascault, J. P. - Die Angew. Makromol. Chem. 230, p.13 (1995).

16. González, F.; Pérez, C.; Ortíz, P. \& Galego, N. Revista C.N.I.C. v. 29, p.119 (1998).

17. Barrett, K. E. J. - J. Appl. Polym. Sci.11, p.1617 (1967).

18. Horie, K.; Hiura, H.; Sawada, M.; Mita, I. \& Kambe, H. - J. Polym. Sci. Part A-1, 8, p.1357 (1970).

Recebido: 13/09/00

Aprovado: 16/11/00 